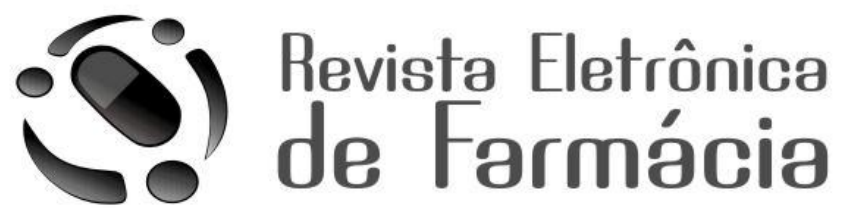

REF - ISSN 1808-0804 Vol. IX (2), 49 - 60, 2012.

\title{
A IMPORTÂNCIA DA RELAÇÃO FARMACÊUTICO - PACIENTE: PERCEPÇÕES DOS IDOSOS INTEGRANTES DA UNATI (UNIVERSIDADE ABERTA À TERCEIRA IDADE) SOBRE A ATUAÇÃO DO FARMACÊUTICO
}

THE IMPORTANCE OF THE RELATIONSHIP PHARMACIST-PATIENT: PERCEPTIONS OF ELDERLY MEMBERS OF UNATI (UNIVERSIDADE ABERTA À TERCEIRA IDADE) ON THE ROLE OF THE PHARMACIST

LA IMPORTANCIA DE LA RELACIÓN FARMACÉUTICO - PACIENTE: PERCEPCIÓN DE LOS MIEMBROS CON EDAD AVANZADA DE LA UNATI (UNIVERSIDADE ABERTA À TERCEIRA IDADE) ACERCA DE LA ACTUACIÓN DEL FARMACÉUTICO

\footnotetext{
Renato Dias D'Andréa ${ }^{1}$, Gisele Paula da Silva ${ }^{2}$, Luciene Alves Moreira Marques $^{3}$, Ricardo Radighieri Rascado ${ }^{4}$

${ }^{1}$ Farmacêutico pela Universidade Federal de Alfenas-MG

${ }^{2}$ Farmacêutica pela Universidade Federal de Alfenas-MG

${ }^{3}$ Doutoranda, Coordenadora do Projeto e Professora da Faculdade de Ciências

Farmacêuticas da Universidade Federal de Alfenas-MG

${ }^{4}$ Doutor, Vice-Coordenador do Projeto e Professor da Faculdade de Ciências

Farmacêuticas da Universidade Federal de Alfenas-MG.
} 
D’Andréa, R.D. da Silva,G.P. Marques, L.A.M. Rascado, R.R. Revista Eletrônica de Farmácia Vol. IX (2), 49 - 60, 2012.

\section{Recebido em 14/11/2011, Aceito em 24/04/2012.}

RESUMO: Em Atenção Farmacêutica, é essencial desenvolver habilidades de comunicação com os pacientes para alcançar melhores resultados com as intervenções propostas. Essa prática é fundamental, pois a relação farmacêutico-paciente implica principalmente em confiança contribuindo para o sucesso do Seguimento Farmacoterapêutico. Desta forma, o objetivo deste trabalho foi conhecer a percepção do paciente sobre o farmacêutico para que se possa desenvolver com base nas necessidades dos pacientes, uma relação farmacêutico-paciente favorável. Foram entrevistados 53 idosos por meio de um instrumento utilizado para avaliar a percepção do paciente acerca do farmacêutico. Aproximadamente $86,79 \%$ eram do sexo feminino. A média de idade entre os idosos foi de 65,82 anos. Em relação à ocupação, constatou-se que $43,39 \%$ são aposentados, $41,51 \%$ do lar, $1,89 \%$ estão desempregados e $3,78 \%$ são comerciantes. Observou-se, pelas respostas, que os idosos valorizam o farmacêutico e almejam que ele tenha uma conduta efetiva de um agente de saúde. Ademais, os idosos concordam com a importância do farmacêutico e querem que este exerça efetivamente o seu papel - o de orientador do tratamento.

PALAVRAS-CHAVE: Idoso, Atenção Farmacêutica, comunicação.

ABSTRACT: In pharmaceutical care, is essential to develop communication skills with patients to achieve better results with the proposed interventions. This practice is essential, because the pharmacist-patient relationship requires trust mainly contributing to the success of the Follow-Pharmaco. Thus, the objective of this study was to assess the patient's perception about the pharmacist in order to develop, based on the needs of patients, a pharmacist-patient relationship in favor. 53 elderly were interviewed through a questionnaire used to assess the patient's perception about the pharmacist. Approximately $86.79 \%$ were female. The average age among the elderly was 65.82 years. Regarding occupation, it was found that $43.39 \%$ are retired, $41.51 \%$ of the home, $1.89 \%$ are unemployed and $3.78 \%$ are traders. It was observed, thus, that older people value the pharmacist and crave it has an effective conduct of a health care provider. Besides the elderly agree with the importance of the pharmacist and want this exercise effectively its role - that of guiding the treatment. 
D’Andréa, R.D. da Silva,G.P. Marques, L.A.M. Rascado, R.R. Revista Eletrônica de Farmácia Vol. IX (2), 49 - 60, 2012.

KEY WORDS: Elderly, Pharmaceutical care, communication.

RESUMEN: En Atención Farmacéutica es esencial desarrollar habilidades de comunicación con los pacientes para lograr mejores resultados con las intervenciones propuestas. Esta práctica es importante, pues la relación farmacéutico-paciente debe basarse principalmente en confianza contribuyendo para el suceso del seguimiento farmacoterapéutico. El objetivo de este trabajo fue evaluar la percepción del paciente acerca del farmacéutico para que se pueda desarrollar con base en las necesidades de los pacientes, una relación farmacéutico-paciente favorable. Fueron encuestados 53 personas con edad avanzada a traves de una encuesta utilizada para evaluar la percepción del paciente acerca del farmacéutico. Cerca de $87 \%$ son mujeres. El promedio de edad fue de 65,82 años. En términos de ocupación, se encontró que el $43,39 \%$ son jubilados, $41,51 \%$ hacen tareas de su casa, $1,89 \%$ no poseen empleo y $3,78 \%$ son comerciantes. Se observa que las personas con edad avanzada aprecian el farmacéutico y les gustaría que él tuviese una conducta efectiva de un agente de salud. Por otra parte, las personas con edad avanzada concuerdan con la importancia del farmacéutico y les gustaria que él desarrollase efectivamente su papel- aquel de orientador del tratamiento.

PALABRAS CLAVE: Anciano; Atención Farmacéutica; comunicación.

\section{INTRODUÇÃO}

É essencial desenvolver
habilidades de comunicação com os pacientes em Atenção Farmacêutica para alcançar melhores resultados com as intervenções propostas. Além disso, a prática da Atenção Farmacêutica está baseada na interação com considerável heterogeneidade de indivíduos, incluindo, pacientes, familiares, profissionais de saúde e outros farmacêuticos. Esta interação social tem sido destacada como a maior indutora da satisfação do paciente em relação aos serviços de saúde ${ }^{(1)}$.

Segundo Lyra Júnior ${ }^{(2)}$, "a comunicação é um instrumento essencial no trabalho do farmacêutico e na promoção da saúde". A escuta ativa é condição essencial para a boa comunicação do farmacêutico, pois the permite entender a realidade do paciente. A partir daí, o farmacêutico 
D'Andréa, R.D. da Silva,G.P. Marques, L.A.M. Rascado, R.R. Revista Eletrônica de Farmácia Vol. IX (2), 49 - 60, 2012.

identifica os pontos chave ou os problemas que mais preocupam o paciente e faz uma análise da situação, com a fundamentação teórica dos problemas identificados. Então, o farmacêutico poderá elaborar hipóteses de solução dos problemas, mas com um plano de cuidados. A partir deste, ele poderá aplicá-las à realidade do paciente, por meio das intervenções farmacêuticas.

Neste sentido, o diálogo facilita o estabelecimento das relações pacientefarmacêutico, num processo simétrico de troca de informações. Significa dizer que $o$ conhecimento científico do farmacêutico não é mais importante que o conhecimento empírico adquirido pela vivência do paciente. Eles são complementares. $O$ paciente passa a cuidar melhor de si quando se sente respeitado e toma consciência de sua importância como agente da própria saúde, e isso têm um efeito positivo direto sobre a sua saúde $e^{(2)}$.

$$
\text { Rantucci }^{(3)} \text { distingue dois }
$$
modelos de abordagem ao paciente: modelo médico e o modelo de ajuda. 0 modelo médico é o tradicional em que o paciente é um agente passivo, ao passo que no modelo de ajuda o paciente tem um papel ativo, desenvolvendo-se a confiança baseada na relação profissional. Este modelo é fundamental na Atenção Farmacêutica, pois a relação farmacêutico-paciente que implica principalmente em confiança é fundamental para o sucesso do Seguimento Farmacoterapêutico.

O processo de Seguimento Farmacoterapêutico consiste em um processo de ajuda e para conclui-lo, a relação farmacêutico-paciente deve ser de mútua confiança. É necessário que o farmacêutico demonstre interesse pelo paciente, com a finalidade de que este compreenda que, as perguntas que são realizadas, redundarão em seu próprio benefício $^{(4)}$.

Carl Rogers ressalta a importância da construção de uma relação de confiança através da aceitação positiva incondicional, empatia, e congruência. Todo o processo terapêutico se desenvolve a partir do princípio de que em cada um existe o potencial necessário para administrar bem sua própria vida. Conseqüentemente, o paciente deixa de ser um sujeito passivo e passa a ser agente do seu próprio tratamento. Ao invés de só esperar os cuidados dos outros, ele é estimulado a cuidar de si mesmo ${ }^{(5)}$.

\section{OBJETIVO}

Desta forma, o objetivo deste trabalho foi conhecer a percepção do 
D’Andréa, R.D. da Silva,G.P. Marques, L.A.M. Rascado, R.R. Revista Eletrônica de Farmácia Vol. IX (2), 49 - 60, 2012.

idoso integrante da Universidade Aberta à Terceira Idade (UNATI) frente ao farmacêutico para que se possa desenvolver uma relação farmacêuticopaciente favorável que permita o desenvolvimento efetivo do Seguimento Farmacoterapêutico aprimorando sua habilidade de comunicação segundo as necessidades apresentadas pelo idoso.

\section{MATERIAL E MÉTODOS}

O trabalho foi realizado na Farmácia-Escola da Universidade Federal de Alfenas, MG (UNIFAL). Este trabalho é parte de um Projeto de Extensão: Atenção Integral à saúde do idoso integrante da UNATI, realizado no período de março de 2006 a novembro de 2008.

O estudo utilizado foi do tipo exploratório e qualitativo, no qual foram entrevistados 53 idosos de ambos os sexos, com idade superior a 50 anos (idade mínima necessária para o ingresso na UNATI). O critério para inclusão no estudo foi: estar matriculado em pelo menos uma atividade promovida pela UNATI.

Todos os sujeitos, que aceitaram participar do Projeto, assinaram o Termo de Consentimento Pós Informado. Os idosos foram atendidos na sala de Atenção Farmacêutica da
Farmácia-Escola da UNIFAL e responderam 2 instrumentos. $\mathrm{O}$ primeiro continha questões sobre sua saúde e medicamentos utilizados (para realização do Seguimento Farmacoterapêutico). No segundo, os pacientes responderam a um questionário composto por 2 partes, sendo a primeira para recolher dados gerais (baseado na Metodologia Dáder ${ }^{(20)}$ ) e a segunda para avaliar como se estabelece a relação farmacêutico-paciente em uma farmácia específica (Farmácia-Escola). $\mathrm{O}$ instrumento utilizado para conhecer a percepção do paciente acerca do farmacêutico foi baseado no trabalho monográfico de DUQUE \& LOURENÇO, $2006^{(6)}$.

Considerando que, a maioria dos dados coletados eram do tipo qualitativo, os mesmos foram organizados por categorias para facilitar a interpretação dos resultados. Foram realizados, primeiramente momentos de leitura livre dos dados para um conhecimento geral do resultado e, posteriormente, buscou-se identificar os temas mais significativos e recorrentes. A partir dessa identificação, foi realizada análise das respostas dos idosos, buscando aumentar a compreensão sobre o tema. 
D'Andréa, R.D. da Silva,G.P. Marques, L.A.M. Rascado, R.R. Revista Eletrônica de Farmácia Vol. IX (2), 49 - 60, 2012.

\section{RESULTADOS}

Aproximadamente $86,79 \%$ eram do sexo feminino. A média de idade foi de 65,82 anos. $43,39 \%$ são aposentados, $41,51 \%$ do lar, $1,89 \%$ estão desempregados e $3,78 \%$ são comerciantes (Figura 1).

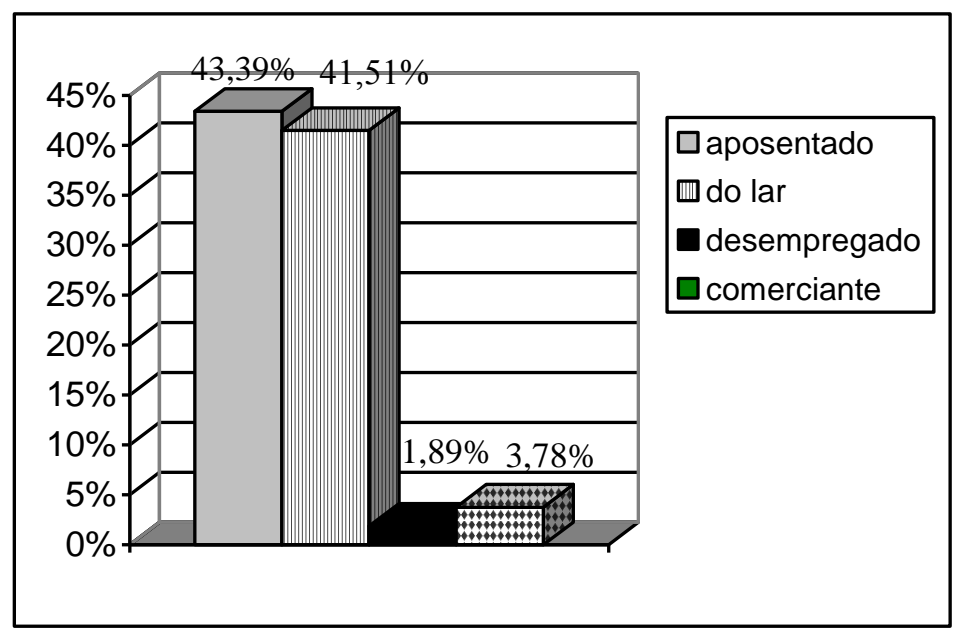

Figura 1: Ocupação dos pacientes participantes do projeto.

Ao serem questionados sobre aspectos relacionados ao farmacêutico, a maioria respondeu que sabe quais as atribuições do farmacêutico e que acha importante a presença dele na farmácia (Tabela 1).

Tabela 1: Perguntas objetivas sobre a percepção do farmacêutico pelo paciente.

\begin{tabular}{|l|c|c|}
\hline \multicolumn{1}{|c|}{ Perguntas } & Sim & Não \\
\hline $\begin{array}{l}\text { Você sabe quais são as atribuições (funções) de um } \\
\text { farmacêutico? }\end{array}$ & $66 \%$ & $34 \%$ \\
\hline $\begin{array}{l}\text { Para você é importante a presença do farmacêutico na } \\
\text { farmácia? }\end{array}$ & $96 \%$ & $4 \%$ \\
\hline $\begin{array}{l}\text { Você já foi atendido por um farmacêutico em outros } \\
\text { estabelecimentos? }\end{array}$ & $47 \%$ & $53 \%$ \\
\hline
\end{tabular}

Ao serem indagados sobre o que esperam do farmacêutico, as respostas mais freqüentes foram:
"Orientação no uso de medicamentos";

"Indicação de medicamentos para males menores"; 
D’Andréa, R.D. da Silva,G.P. Marques, L.A.M. Rascado, R.R. Revista Eletrônica de Farmácia Vol. IX (2), 49 - 60, 2012.

"Que ele esteja presente na farmácia; seja dedicado e responsável". Ao serem indagados se 0 farmacêutico atende suas necessidades, algumas das respostas foram:

"Sim, porque tira as dúvidas sobre o tratamento";

"Sim, porque orienta com paciência";

"Sim, porque tem mais conhecimento e passa confiança".

\section{DISCUSSÃO}

A média de idade encontrada entre os idosos foi de 65,82 anos. No Brasil há dispositivos legais que consideram idosos os indivíduos com idade igual ou superior aos 60 anos, quais sejam: o Decreto no 1948/96, que regulamenta a Lei no 8842/94, estabelece a Política Nacional do Idoso $^{(7)}$, além disso o país possui o Estatuto do Idoso, Lei no 10.741 , de $1^{\circ}$ de outubro de $2003^{(8)}$. Apesar de alguns órgãos reconhecerem que aos 60 anos o indivíduo seja considerado idoso, alguns autores brasileiros postulam que o idoso é aquele com 65 anos ou mais, tanto para fins sociodemográficos, quanto para abordagem relacionada à farmacoterapia ${ }^{(9-10)}$. Entretanto, para ingresso na UNATI, a pessoa deve ter 50 anos ou mais. Isso se deve ao fato
Também foi solicitado aos idosos que completassem a seguinte frase: Um bom farmacêutico é aquele que...

"Orienta bem",

"Está sempre na farmácia",

"Dá atenção",

"Transmite confiança e conhecimento". de que há uma procura significativa pelas atividades da UNATI por pessoas que ainda não completaram 60 anos. Além disso, a participação precoce nas atividades pode contribuir para uma melhor qualidade de vida.

Quase $100 \%$ dos idosos afirmaram que é importante a presença de um farmacêutico na farmácia (Tabela 1). Para Oliveira(11), o farmacêutico é capaz de desenvolver atividades de Assistência Farmacêutica em qualquer âmbito de atuação, devendo ter, além da formação técnica, habilidades de comunicação para desenvolver seu papel de educador junto à população quanto aos cuidados com a saúde e o uso racional de medicamentos.

Ao serem indagados sobre o que esperam do farmacêutico, as respostas mais freqüentes foram: orientação no uso de medicamentos; indicação de 
D’Andréa, R.D. da Silva,G.P. Marques, L.A.M. Rascado, R.R. Revista Eletrônica de Farmácia Vol. IX (2), 49 - 60, 2012.

medicamentos para males menores; esteja presente na farmácia; seja dedicado e responsável. Segundo Campmany ${ }^{(12)}$, o farmacêutico deve assegurar que $o$ paciente tenha e entenda a informação necessária para utilizar o medicamento que lhe é entregue de maneira segura e eficaz. Nesse sentido, Pepe \& Castro $^{(13)}$ ressaltam que a informação prestada ao paciente no ato da dispensação do medicamento é tão ou mais importante que o produto por ele recebido.

O aconselhamento é um processo de escuta ativa, individualizado e centrado no paciente. Pressupõe a capacidade de estabelecer uma relação de confiança entre os atores envolvidos, visando ao resgate dos recursos internos da pessoa atendida, para que ela mesma tenha possibilidade de reconhecer-se como sujeito de sua própria saúde e transformação ${ }^{(14)}$.

Ao serem indagados se 0 farmacêutico atende suas necessidades, as respostas foram: sim, porque tira as dúvidas sobre o tratamento; porque orienta com paciência; porque tem mais conhecimento e passa confiança. Além de problemas de caráter geral característicos da idade, os idosos encontram-se, freqüentemente, também sujeitos a problemas decorrentes da baixa auto-estima, da solidão ou da marginalização circunstancial $^{(15)}$. Tal aspecto justifica a crucialidade, conforme a ótica dos idosos, da atuação do farmacêutico de forma pacienciosa e dedicada, de modo a oferecer não apenas conhecimento específico, mas valores humanos e sociais que almejem a confiança do profissional também como pessoa e amigo.

\begin{tabular}{rrrr} 
Quando & \multicolumn{2}{c}{ solicitou-se } & que \\
completassem a frase: Um bom \\
farmacêutico é aquele que...
\end{tabular}
respostas mais freqüentes foram: Orienta bem, está sempre na farmácia, dá atenção, transmite confiança e conhecimento dentre outras respostas.

A posição que o farmacêutico ocupa na escala de valores do paciente é decisiva para determinar a maior ou menor adesão do paciente ao tratamento prescrito, como também para delinear a ótica dos pacientes idosos sobre o profissional. Assim, as relações terapêuticas devem ser construídas com fundamento no respeito mútuo, honestidade, autenticidade, comunicação aberta, cooperação, empatia, sensibilidade, paciência, compreensão, confiança, confidência, colaboração com o paciente e demais profissionais, oferecer segurança, promoção da 
D’Andréa, R.D. da Silva,G.P. Marques, L.A.M. Rascado, R.R. Revista Eletrônica de Farmácia Vol. IX (2), 49 - 60, 2012.

independência do paciente, enxergá-lo como uma pessoa, dar-Ihe prioridade e apoio, a fim de promover, além do sucesso do tratamento, a valorização do profissional como agente de saúde ${ }^{(16)}$.

O conhecimento adquirido pelo paciente, algumas vezes, o faz ter uma percepção dos seus problemas relacionados aos medicamentos. Portanto, o farmacêutico deve atuar não somente como um clínico, mas também deve ser um educador em saúde e um amigo do paciente. Neste sentido, é importante questionar sempre os pacientes, para que esgotem todas as suas dúvidas, pois todas as perguntas são importantes para o uso correto dos medicamentos e para a manutenção da saúde ${ }^{(17)}$.

Cipolle, Strand e Morley ${ }^{(16)}$ descrevem que o cuidado farmacêutico é um exercício profissional generalista, relacionado ao cuidado com o paciente em problemas relacionados com os medicamentos. Alguns problemas relacionados com os medicamentos são bem simples e fáceis de serem solucionados, enquanto outros são complexos e podem levar tempo para sua resolução.

Eis então a importância do farmacêutico de possuir habilidades de comunicação, valendo-se de estratégias pedagógicas para estabelecer uma comunicação extremamente proveitosa, e conseqüentemente prover ao paciente, através do processo de Atenção Farmacêutica, todas as informações necessárias sobre o uso do medicamento capaz de comprometê-lo efetivamente com o cumprimento do seu tratamento ${ }^{(18)}$.

Segundo Hammond et al. ${ }^{(19)}$, a comunicação direta com os pacientes é imperativa para que os farmacêuticos possam influir no sucesso da terapia medicamentosa. Esta relação contribui para melhor desempenho do serviço, além de possibilitar o registro de dados do paciente, tais como evolução do tratamento, problemas ocorridos e resultados laboratoriais. Consiste em um ato profissional importante, que envolve questões técnicas, humanas e éticas.

A prática médica de não informar ao paciente os motivos de sua escolha por um ou outro procedimento, mantém o paciente numa posição infantil em relação à sua autoridade. Pode-se observar neste trabalho, que os idosos não valorizam este tipo de conduta. Os idosos querem receber orientações sobre o seu tratamento, solucionar suas dúvidas em relação ao tratamento e mais que isso, querem ser bem atendidos e respeitados. 
D'Andréa, R.D. da Silva,G.P. Marques, L.A.M. Rascado, R.R. Revista Eletrônica de Farmácia Vol. IX (2), 49 - 60, 2012.

\section{CONCLUSÕES}

Os resultados obtidos

demonstram que os idosos confiam no farmacêutico para solucionar seus problemas de saúde ou esclarecer dúvidas referentes aos medicamentos. Estes achados permitem elaborar estratégias de uma melhor comunicação com os idosos a fim de aumentar a efetividade da dispensação de medicamentos e do seguimento farmacoterapêutico.

\section{Agradecimentos}

Ao Proext MEC/SESU pelo apoio financeiro e à UNIFAL-MG.

\section{REFERÊNCIAS}

1. Lyra júnior DP, Amaral RT, Abriata JP, Pelá IR. A satisfação como resultado de um programa de atenção farmacêutica para pacientes idosos, em Ribeirão Preto (SP)Brasil. Seguimiento Farmacoterapéutico. 2004; 3(1): 30-42.

2. Lyra júnior DP. Comunicação paciente/ farmacêutico: um instrumento libertário e essencial no trabalho do profissional e na promoção da saúde. Pharmacia Brasileira. $2005 ; 1(6): 10$.

3. Rantucci MJ. Guia de consejo del farmacéutico al paciente. Barcelona MassonWilliams \& Wilkins. 1998.

4. Caelles N, Ibáñez J, Machuca M, Martínez-romero F, Faus MJ. Entrevista farmacéutico-paciente en el Programa Dáder de seguimiento farmacoterapéutico. Pharmaceutical Care España. 2002; 4: 55-59.

5. Silva LC. Relação Farmacêutico-Paciente. In: Marques LAM, editors. Atenção Farmacêutica em distúrbios maiores.1ed. Medfarma; 2009.p 67-116.

6. Duque DCC, Lourenço, EB. Curso de Especialização em Atenção Farmacêutica da UNIFAL-MG [monography]. 2006. 
D’Andréa, R.D. da Silva,G.P. Marques, L.A.M. Rascado, R.R. Revista Eletrônica de Farmácia Vol. IX (2), 49 - 60, 2012.

7. Lei N. 8.842 de 4 de janeiro de 1994. Dispõe sobre a Política Nacional do Idoso, cria o Conselho Nacional do Idoso e dá outras providências. Diário Oficial da União (Brasília). 2009 Ago 11.

8. Lei n. 57, de 23 de setembro de 2003 - Dispõe sobre o Estatuto do Idoso e dá outras providências. Diário Oficial da União (Brasil); 2009 Ago 12.

9. Bisson MP. Farmácia Clínica e Atenção Farmacêutica. Seguimento de pacientes Idosos, 2 ed. São Paulo; 2007. p. 208.

10. Carvalho MFC. A polifarmácia em idosos no município de São Paulo - Estudo SABE - Saúde, Bem-estar e Envelhecimento. [dissertation]. São Paulo/SP. Biblioteca Digital de Teses e Dissertações - USP, 2008.

11. Oliveira MJL. Assistência Farmacêutica: a percepção dos gestores e profissionais de saúde em São Luís (MA). [dissertation]. Porto Alegre/RS. Programa de Pós-Graduação em Ciências Farmacêuticas, UFRGS, 2004.

12. Campmany ME. Identificación Del paciente y estratégias de comunicación. Âmbito Farmacêutico. Dispensación Activa. 2006; 25(3):78-84.

13. Pepe VLE, Castro CGS. A interação entre prescritores, dispensadores e pacientes: Informação compartilhada como possível beneficio terapêutico. Cadernos de Saúde Pública. 2000; 16: 815-822.

14. Ministério da Saúde; Coordenação Nacional de DST e Aids - Aconselhamento em DST, HIV e Aids: Diretrizes e Procedimentos Básicos.Brasília (Brasil); 1997.

15. Ranelli, PL, Coward RT. Communication between pharmacists and patients: The role of place of residence in determining the expectations of older adults.

Pharmacotherapy.1997; 17:148-162.

16. Cipolle RJ, Strand LM, Morley PC. O exercício do cuidado farmacêutico. Conselho Federal de Farmácia [Internet]. 2006 [cited 2009 ago 26]; 378 p. 
D’Andréa, R.D. da Silva,G.P. Marques, L.A.M. Rascado, R.R. Revista Eletrônica de Farmácia Vol. IX (2), 49 - 60, 2012.

17. Possamai FP, Dacoreggio MS. A habilidade de comunicação com o paciente no processo de atenção farmacêutica. Trab. Educ. Saúde.2008;5(3):473-490.

18. Marin N.. Assistência farmacêutica para gerentes municipais. Opas/OMS, 2003.

19. Hammond RW, Schwartz AH, Campbell MJ. Collaborative drug therapy management by pharmacists. Pharmacoterapy. 2003; 23(9):1210- 1225.

20. Dader MJF, Romero FM. La atención farmacéutica em farmacia comunitaria:

evolución de conceptos, necesidades de formación, modalidades y estrategias para su puesta en marcha. Pharmaceutical Care España. 1999;1:52-61. 